\title{
FORMAÇÃO DE COMPETÊNCIAS PARA O GERENCIAMENTO EM ENFERMAGEM*
}

Maria de Lourdes de Almeida1, Aida Maris Peres², Elizabeth Bernardino², Marieta Fernandes Santos ${ }^{3}$

${ }^{1}$ Enfermeira. Mestre em Enfermagem. Universidade Federal do Paraná. Curitiba-PR-Brasil.

Enfermeira. Doutora em Enfermagem. Universidade Federal do Paraná Curitiba-PR-Brasil.

${ }^{3}$ Enfermeira. Doutora em Enfermagem. Universidade Estadual do Oeste do Paraná. Foz do Iguaçu-PR-Brasil.

RESUMO: O objetivo deste estudo exploratório-descritivo foi identificar os conhecimentos, habilidades e atitudes apreendidas por 22 egressos de um curso de graduação na temática de Gerenciamento em Enfermagem. O período de coleta de dados foi de fevereiro a abril de 2010; utilizou-se, para a análise dos dados, a técnica do Discurso do Sujeito Coletivo que tem como pressuposto a Teoria da Representação Social. Como resultados surgiram Discursos do Sujeito Coletivo referentes à utilização de instrumentos gerenciais nas ações gerenciais: conhecimentos sobre gerenciamento dos recursos humanos, materiais, físicos e ambientais e do cuidado de enfermagem; habilidades gerenciais como comunicação, educação permanente, tomada de decisão; resolução de problemas e liderança; atitude embasada pelo conhecimento e influenciada pelo papel institucional da Enfermagem. O ensino do gerenciamento na graduação destaca-se pela sustentação que este propicia para o desenvolvimento de conhecimentos, habilidades e atitudes que embasam a prática gerencial em enfermagem. DESCRITORES: Educação em enfermagem; Estudantes de enfermagem; Gerência.

\section{THE TRAINING OF COMPETENCES FOR MANAGEMENT IN NURSING*}

\begin{abstract}
The objective of this exploratory-descriptive study was to identify the knowledge, skills and attitudes learned by 22 graduates from an undergraduate course in the issue of Management in Nursing. The data collection period was February - April 2010; for data analysis it used the technique of the Discourse of the Collective Subject, which is based on Social Representation Theory. Results: Discourses of the Collective Subject appeared referent to the use of managerial instruments in managerial actions: knowledge regarding management of human, material, physical, environmental and nursing care related resources; managerial skills such as communication, continuous education, and decision-making; problem resolution and leadership; and attitude based on knowledge and influenced by the institutional role of nursing. The teaching of management at the undergraduate level stands out through the support which this provides for the development of knowledge, skills and attitudes which form a base for managerial practice in nursing.
\end{abstract}

DESCRIPTORS: Education in Nursing; Student nurses; Management.

\section{FORMACIÓN DE COMPETENCIAS PARA LA AD- MINISTRACIÓN EN ENFERMERÍA}

RESUMEN: El objetivo de este estudio exploratorio descriptivo fue identificar los conocimientos, habilidades y actitudes de 22 egresos de un curso de graduación en la temática de Administración en Enfermeríam. Los datos fueron obtenidos de febrero a abril de 2010; fue utilizada, para el análisis de los datos, la técnica del Discurso del Sujeto Colectivo que tiene como presupuesto la teoría de la Representación Social. Como resultados surgieron Discursos del Sujeto Colectivo referentes a la utilización de instrumentos administrativos en las acciones gerenciales: conocimientos acerca de la administración de los recursos humanos, materiales, físicos y ambientales y del cuidado de enfermería; habilidades administrativas como comunicación, educación permanente, tomada de decisión; resolución de problemas y liderazgo; actitud basada por el conocimiento e influenciada por el papel institucional de la enfermería. La enseñanza de la administración en la graduación se destaca por la sustentación que esta propicia para el desarrollo de conocimientos, habilidades y actitudes que fundamentan la práctica de gerencia en enfermería. DESCRIPTORES: Educación en enfermería; Estudiantes de enfermería; Gerencia.

*Artigo extraído da dissertação de mestrado Gerenciamento em Enfermagem: formação e prática na perspectiva de egressos de uma universidade pública, 2010.

Recebido: $13 / 11 / 2013$ Finalizado: 15/03/2014

Autor correspondente:

Maria de Lourdes de Almeida

Universidade Estadual do Oeste do Paraná

Rua TarquínioJoslimdos Santos, 1300-85870-650-Foz dolguaçu-PR-Brasil

E-mail: m_lourdesdealmeida@yahoo.com.br 


\section{INTRODUÇÃO}

O trabalho do enfermeiro, como uma prática social articulada a outras práticas einstrumento do processo de trabalho em saúde, tem como subdivisão as dimensões cuidar/assistir, administrar/gerenciar, pesquisar e ensinar ${ }^{(1)}$. Frente à versatilidade de atuação profissional, em conjunto à globalização e às mudanças tecnológicas que provocam flexibilidade nos processos de trabalho, são exigidos novos perfis profissionais para acompanhar a lógica deste mercado contemporâneo; desse modo, enfermeiros e outros profissionais de saúde passam a demandar o desenvolvimento de novas competências $^{(2)}$.

Várias são as definições para a palavra competência, mas neste artigo, utilizou-se como referência o conceito que propõe a definição centrada na mudança de comportamento social das pessoas em relação ao trabalho e sua organização. Para o autor, a competência é tomar iniciativa e assumir responsabilidade do trabalhador em cenários profissionais. $\mathrm{O}$ tomar iniciativa é começar algo, uma ação que transforma o que existe, inserir algo novo ${ }^{(3)}$.

Nesta vertente, as políticas educacionais brasileiras apresentam, no texto das Diretrizes Curriculares Nacionais (DCN) para os Cursos de Enfermagem, a descrição da formação do enfermeiro voltada a atender as necessidades sociais da saúde, com o objetivo de favorecer o desenvolvimento de conhecimentos, habilidades e atitudes requeridos para a atuação profissional competente e que subsidie as ações nos diferentes âmbitos profissionais. As DCN consideram que cabe ao enfermeiro a coordenação do processo de cuidar em Enfermagem, nos contextos e demandas de saúde ${ }^{(4)}$.

Nas DCN, das 7 competências gerais exigidas dos profissionais de saúde, dentre esses, o enfermeiro, 6 estão relacionadas ao trabalho gerencial: tomada de decisão, comunicação, liderança, trabalho em equipe, administração e gerenciamento e educação permanente ${ }^{(1)}$. $\mathrm{Na}$ dimensão administrar/gerenciar destacase o gerenciamento em Enfermagem, o qual constitui atividade complexa visto que exige dos profissionais competências cognitivas, técnicas e atitudinais $^{(5)}$.

No atual panorama de formação do enfermeiro, no que diz respeito ao seu processo de ensino- aprendizagem, coloca-se como desafio para a profissão a formação de profissionais competentes e compromissados com a sociedade e com os seus problemas de saúde. A articulação da teoria e da prática pode propiciar, ao futuro trabalhador, uma visão crítica da realidade, considerando a complexidade do indivíduo e o contexto em que este vive e trabalha ${ }^{(6)}$.

O gerenciamento em Enfermagem, no Brasil, é legitimado pela Lei do Exercício Profissional de Enfermagem (Lei n. 7.498/1986), que define como atividades privativas do enfermeiro a direção de órgão de Enfermagem, a organização e a direção do Serviço de Enfermagem e de unidade de Enfermagem. Como uma das dimensões do processo de trabalho do enfermeiro, o gerenciamento requer a mobilização de competências para o exercício das ações gerenciais voltadas para a qualidade do cuidado prestado, e com conexão entre a gerência de nível hierárquico superior e os trabalhadores da base ${ }^{(7)}$.

Esse estudo aborda o a formação de competências para o gerenciamento em Enfermagem, tema relevante dada a importância da sua atuação na atenção à saúde, haja vista que sugere mudanças como forma de propiciar o desenvolvimento de competências gerenciais nos diferentes cenários no mundo do trabalho do enfermeiro. Frente ao exposto, esta pesquisa teve como objetivo identificar quais são os conhecimentos, habilidades e atitudes apreendidas por egressos de um curso de graduação, no que se referiu à temática de Gerenciamento em Enfermagem.

\section{MÉTODO}

Estudo descritivo-exploratório de abordagem qualitativa foi realizado na Universidade Estadual do Oeste do Paraná - Unioeste, Campus de Foz do Iguaçu, na região da Tríplice Fronteira Brasil, Paraguai e Argentina, e teve como população da pesquisa os 59 formandos das turmas de 2007 a 2009 do Curso de Enfermagem do referido campus.

A amostra constituiu-se de 22 egressos de ambos os sexos que se encaixavam nos seguintes critérios de inclusão: ter atuado profissionalmente em alguma área de Enfermagem por tempo igual ou superior a seis meses. Para o cálculo da amostra foi utilizada proporção máxima, com nível de confiança de 95\% e erro de 5\%. 
A coleta de dados ocorreu nos meses de fevereiro a abril de 2010, após validação do instrumento de coleta de dados por meio de pré-teste. Os dados foram coletados por meio de entrevista semiestruturada gravada para aprofundamento das questões relacionadas à formação gerencial dos participantes, após aprovação por comitê de ética (registro n. 0054.0.091.000-09). As questões norteadoras utilizadas para as entrevistas estão abaixo discriminadas em conjunto aos respectivos temas (Quadro 1):

Quadro 1 - Descritivo das questões norteadoras e de seus respectivos temas. Foz do Iguaçu, PR, Brasil, 2010.

\begin{tabular}{|l|l|}
\hline Questão Norteadora & Tema \\
\hline $\begin{array}{l}\text { Em que situações o egresso utiliza os conhecimentos } \\
\text { que adquiriu nas disciplinas de administração no seu } \\
\text { curso de graduação em Enfermagem? }\end{array}$ & Conhecimentos gerenciais requeridos \\
\hline $\begin{array}{l}\text { Para que o egresso utilize esses conhecimentos, que } \\
\text { habilidades gerenciais são requeridas? }\end{array}$ & Habilidades gerenciais requeridas \\
\hline $\begin{array}{l}\text { Quais atitudes relacionadas à gerência são } \\
\text { desempenhadas na prática profissional do egresso? }\end{array}$ & Atitudes gerenciais requeridas \\
\hline
\end{tabular}

Para a análise dos dados utilizou-se da técnica do Discurso do Sujeito Coletivo (DSC) ${ }^{(8)}$. Esta técnica consiste no uso de estratégia discursiva para tornar mais clara uma dada representação social, bem como o conjunto das representações que conforma um dado imaginário; tem como fundamento a teoria da Representação Social e seus pressupostos sociológicos, com a proposta de analisar o material verbal coletado que é extraído dos depoimentos ${ }^{(9)}$.

Em estudos realizados com esta técnica, o pensamento é coletado por meio de entrevistas para que esse possa ser explicitado(8). O significado das opinões coletivas é um processo complexo que exige várias operações em momentos específicos e são realizadas sobre o material verbal que foi coletado no estudo. São quatro as operações realizadas para a construção dos discursos ${ }^{(8-10)}$ :

- Expressões Chaves: primeiramente extraem-se dos depoimentos as Expressões-Chave (E-Ch), que são pedaços selecionados do material da entrevista que foi transcrito, que melhor descrevem o seu conteúdo, ou seja, a sua essência. Os depoimentos coletivos, propriamente ditos, são formados por um conjunto de E-Ch de sentido semelhante ou complementar.

Estes discursos são redigidos na primeira pessoa do singular a fim de evidenciar o pensamento coletivo como um sujeito coletivo de discurso ${ }^{(10)}$. Os indivíduos podem compartilhar ideias semelhantes, mas, quando tem uma opinião individual, transferem apenas uma parte do conteúdo da ideia compartilhada(10).
- Ideias Centrais: a seguir constrõem-se as Ideias Centrais (ICs), que no DSC são equivalentes às categorias. As ICs são fórmulas que sintetizam e descrevem o sentido presente nos depoimentos de cada uma das respostas e, ainda, no conjunto de respostas de diferentes indivíduos, no qual apresentam sentido semelhante ou que os complementam $^{(8-10)}$.

- Ancoragens (ACs): são como as ICs, mas descrevem os valores, as crenças que podem surgir do material verbal das respostas individuais ou que foram agrupadas como afirmações genéricas para enquadrar situações particulares ${ }^{(8-10)}$. Segundo a metodologia do DSC, as ACs só devem ser utilizadas quando é encontrado, no material verbal, marcas discursivas explícitas dessas afirmações genéricas ${ }^{(8)}$, o que não ocorreu nos resultados da presente pesquisa.

- Discurso do Sujeito Coletivo: é o resultado da reunião das E-Ch encontradas nos depoimentos que têm ICs e/ou ACs de sentido semelhante ou complementar. Cada DSC é o resultado da contribuição de certo número de entrevistas ou depoimentos dos indivíduos ${ }^{(8-10)}$.

O DSC é uma soma qualitativa, com agregação dos elementos que o compõem - as E-Ch de respostas semelhantes; não é produto de uma quantidade determinada de iguais, mas semelhantes que individualiza um determinado sentido, uma opinião coletiva que é diferente de outro sentido, outra opinião coletiva que conformará outro discurso. A pessoa coletiva fala como se fosse um indivíduo, um sujeito de discurso que transporta a representação de vários 
indivíduos, emergindo uma opinião coletiva qualitativa e quantitativa ${ }^{(10)}$.

Os autores definem o DSC como uma forma que não é matemática, nem metalinguística para representar e produzir o pensamento de dada coletividade. Um sujeito coletivo, no DSC, constitui tentativa de reconstituir um sujeito coletivo que, enquanto pessoa coletiva esteja, ao mesmo tempo, falando como se fosse indivíduo, isto é, como um sujeito de discurso natural, mas veiculando uma representação com conteúdo ampliado ${ }^{(8)}$.

\section{RESULTADOS}

A caracterização dos sujeitos apontou que, dos 22 entrevistados, 14 (63,6 \%) são do sexo feminino e 8 (36,4\%) sujeitos do sexo masculino. A maioria dos egressos concluiu o curso no ano de 2008. Sobre o local de residência, 20 egressos (90\%) são do município de Foz do Iguaçu Paraná, um (5\%) reside em Medianeira - Paraná, e um (5\%) em Belém - Pará.

$\mathrm{Na}$ análise dos depoimentos foram utilizadas as E-Ch e ICs e o discurso síntese como figuras metodológicas de linguagem para a construção do $D S C^{(9)}$, com exceção da $A C$, que não foi identificada, no material das entrevistas, marca discursiva explícita de afirmações genéricas que configurassem ideologias, valores ou crenças. Os temas e as ICs que emergiram dos depoimentos estão apresentados no Quadro 2.

A partir da análise da IC 1, emergem conhecimentos de temas específicos, como: auditoria, mapeamento de processos, planejamentos de reuniões, relatórios, sistemas de informação, teorias administrativas e normas e rotinas. Isso demonstra a utilização dos conhecimentos gerenciais nas ações assistenciais, ensino e trabalho em equipe.

Na sequência da análise deste tema, a IC 2 evidenciou a percepção dos egressos de que, para gerenciar a equipe de Enfermagem, são mobilizados conhecimentos sobre recrutamento,

Quadro 2 - Temas e ideias centrais. Foz de Iguaçu, PR, Brasil, 2010.

\begin{tabular}{|l|l|}
\hline TEMAS & IC \\
\hline $\begin{array}{l}\text { Conhecimentos gerenciais requeridos para a prática } \\
\text { profissional }\end{array}$ & $\begin{array}{l}\text { 1: Utilização dos conhecimentos em situações } \\
\text { diversas } \\
\text { 2: Utilização dos conhecimentos nas situações de } \\
\text { gerenciamento da equipe }\end{array}$ \\
\hline $\begin{array}{l}\text { Habilidades gerenciais requeridas para a prática } \\
\text { profissional }\end{array}$ & $\begin{array}{l}\text { 3: Habilidades gerenciais como: comunicação, } \\
\text { educação permanente, tomada de decisão } \\
\text { e resolução de problemas, pessoais e } \\
\text { autoconhecimento e de liderança }\end{array}$ \\
\hline Atitudes gerenciais requeridas na prática profissional & $\begin{array}{l}\text { 4: Embasada pelo conhecimento e influenciada } \\
\text { pelo papel institucional da Enfermagem } \\
\text { 5: Gerenciamento do tempo, de liderança e de } \\
\text { recursos humanos }\end{array}$ \\
\hline
\end{tabular}

seleção e treinamento e avaliação de desempenho, dimensionamento de pessoal e distribuição de atividades, escalas, liderança, trabalho em equipe e relacionamento interpessoal.

Sobre o tema Habilidades gerenciais requeridas para a prática profissional a IC 3 apontou habilidades diversas que o enfermeiro necessita possuir para o desempenho gerencial.

Surgiu a referência sobre habilidade de marketing pessoal, relacionado à visibilidade do enfermeiro. A base para tomada de decisão, segundo os egressos, é a competência técnica, saber fazer permite o levantamento de problemas, proposição de metodologia e avaliação dos resultados propostos.

No último tema investigado relacionado às Atitudes gerenciais requeridas na prática profissional na IC 4, os sujeitos apreenderam a dimensão atitudinal como a mais complexa e como o grande desafio para a atuação do enfermeiro. O discurso dos egressos dessa IC apontou que nas situações de ensino, o sujeito não consegue desenvolver as atitudes, frente a isto precisa fundamentar a prática profissional 
com conhecimentos e habilidades. Outro fator importante no discurso dos sujeitos desta IC é o papel institucional da Enfermagem, que exerce influência sobre as atividades do enfermeiro.

$\mathrm{Na}$ IC 5, o gerenciamento do tempo aparece como forma de cumprir as tarefas e dar conta das atividades de planejamento, organização e direção que o enfermeiro desenvolve. O egresso relata sua dificuldade em assumir o cuidado direto e valoriza o enfoque de uma liderança voltada a controlar o cuidado prestado pela equipe. Além disso, a IC 5 mostra as atitudes dos enfermeiros no gerenciamento de recursos humanos na Enfermagem. Esses têm enfoque no desenvolvimento de habilidades relacionadas a tarefas gerenciais, mas falta reflexão e planejamento já que as atitudes evidenciam um olhar tarefeiro e imediatista na sua prática.

\section{DISCUSSÃO}

Conforme evidenciado nos resultados, o enfermeiro mobiliza conhecimentos de temas específicos da administração na sua prática profissional, que por não terem sido abordados como temas centrais durante sua formação, necessitam de aprofundamento em situações específicas, obrigando o egresso a buscá-los. Para suprir essa lacuna, uma política de educação permanente institucional possivelmente poderia favorecer o aprofundamento dos temas de acordo com as demandas de cada ambiente.

Em pesquisa realizada sobre a percepção de enfermeiros em relação à disciplina Administração aplicada à Enfermagem na prática profissional, esses profissionais reconhecem a importância desta dimensão e a necessidade de aprofundamento no seu ensino. Consideraram que o preparo e a segurança permitem o desenvolvimento de uma atitude reflexiva para enfrentar situações de trabalho, e o estágio realizado na disciplina foi percebido como idealizado $^{(11)}$.

$\mathrm{Na}$ mobilização de conhecimentos sobre gerenciamento de pessoas, um estudo realizado com enfermeiros de uma unidade hospitalar relata que as atividades administrativas mais desenvolvidas foram: passagem de plantão, escala diária e tarefas de funcionários, e gerenciamento de exames. Como atividades assistenciais, referiram ações ligadas ao relacionamento com a equipe multiprofissional e com a implementação da Sistematização da Assistência de Enfermagem ${ }^{(12)}$. Não foram percebidas pelos participantes do estudo citado dicotomia entre as atividades gerenciais e assistenciais, mas as assistenciais foram relatadas como as mais prazerosas, o que pode evidenciar as dificuldades no desenvolvimento de ações gerenciais voltadas à equipe de Enfermagem.

Outros conhecimentos apontados na IC 2 e que servem de base para o gerenciamento de pessoas são pautados nas legislações referentes ao gerenciamento de recursos humanos, como as leis trabalhistas, Lei de Exercício Profissional de Enfermagem, normas e rotinas em Enfermagem, dimensionamento de pessoal, que compõem um corpo de conhecimentos que sustentam a tomada de decisão dos enfermeiros em relação ao gerenciamento de pessoas.

Nas questões de gerenciamento da assistência, surgiu o discurso sobre conhecimentos relacionados à administração do tempo que permitem priorizar e delegar atividades e planejar a assistência. Este resultado é corroborado por um estudo realizado com enfermeiras de uma instituição hospitalar em que a falta planejamento resulta na administração inadequada de seu tempo de trabalho e assim não conseguem realizar de todas as atividades demandadas no seu turno de trabalho. Para os participantes do estudo citado, o seu trabalho isso pode ocasionar trabalho repetitivo e sem reflexão com consequência na falta de otimização do tempo disponível ${ }^{(13)}$.

Na sequência da discussão, observa-se que na IC 3 o enfermeiro utiliza a habilidade de comunicação com o usuário e com a equipe no sentido de administrar conflitos, orientar e intervir na condição de elo institucional. Para o fortalecimento de tais habilidades, busca-se no contexto contemporâneo, um diferencial na gestão de pessoas, que pode ser facilitado por meio de estratégias para a superação da visão tradicional da administração de recursos humanos, que ainda percebe as pessoas como recursos semelhantes aos demais recursos da organização(14).

Para um novo cenário na gestão das pessoas necessita-se mudança na forma da gestão das organizações atuais, para que estas tendam a ser mais participativas, democráticas e assumam 
perspectivas de maior diversidade. Essas mudanças devem ser bem vistas e aceitas, pois crescimento, comunicação, caminhos para acordos, colaboração e corresponsabilidade são palavras que auxiliarão a atacar problemas e não pessoas ${ }^{(15)}$.

A temática liderança é citada também como uma habilidade gerencial. A relevância dessa habilidade foi abordada num estudo em que os autores perceberam que esses enfermeiros vêem a liderança como um processo de interação entre grupos de pessoas com objetivos comuns. Para os enfermeiros que participaram de tal estudo, a competência pode ser desenvolvida, melhorada e construída ao longo da formação ${ }^{(16)}$.

$\mathrm{Na}$ prática profissional da Enfermagem, a habilidade que emerge na IC 3, voltada ao processo de tomada de decisão, tem sido pautada na experiência do cotidiano, isto é, no conhecimento empírico que pode resultar em decisões acertadas ou em erros. Autoras da área de administração sugerem que, para a tomada de decisão com qualidade, além de serem mais assertivos, os gestores necessitam de um método a ser seguido ${ }^{(1)}$.

Sendo assim, uma sistematização da tomada de decisão é proposta a partir de uma sequência que se inicia com a identificação do problema e reunião de dados, seguida da identificação de causas e consequências do problema e, a partir disto, com a proposição de soluções alternativas para serem implementadas. Após é realizada a avaliação dos resultados e, caso seja necessário, inicia-se novamente esta sequência. Ao ser utilizada pelos enfermeiros, esta sistematização pode desencadear decisões mais assertivas na sua prática profissional ${ }^{(17)}$.

No campo atitudinal, no que se refere à IC 4, percebe-se que as atitudes se entrelaçam com os conhecimentos e habilidades e dependem do indivíduo, vêm depois dele, respaldadas nos conhecimentos e habilidades. Sobre isso, autores $^{(18)}$ apontam que ensinar conteúdos científicos, procedimentais e conceituais é mais fácil; o atitudinal é mais complexo e requer que os docentes tenham consenso do que seriam as atitudes a serem desenvolvidas, e tê-las como objetivos de aprendizagem no curso de graduação, na busca de construir instrumentos adequados de avaliação.

Para tanto, o campo de prática constitui momento ímpar e fértil para trabalhar conteúdos atitudinais. O principal conteúdo trabalhado na graduação, que contribui com o campo atitudinal, é a tomada de decisão, isto não significa que outros conteúdos não permeiem a atitude. Para que os conteúdos contribuam nesta construção da atitude, é necessário que as metodologias sejam ativas e propiciem experiências e vivências de situações de realidade ou fictícia para fortalecê-las.

Para corroborar com este resultado, outro estudo realizado sobre a vivência de acadêmicos de enfermagem nas disciplinas de Administração de Enfermagem evidenciou que a recepção do acadêmico no campo da prática pelos trabalhadores dos serviços é importante para o desenvolvimento de aulas práticas, o que facilita a mobilização de conhecimentos aprendidos na teoria, sua relação com a prática e o desenvolvimento do senso critico no acadêmico(19).

$\mathrm{Na}$ continuidade de análise dessa temática, na IC 5, outras atitudes gerenciais na prática profissional do enfermeiro trazem o seu trabalho sob o enfoque produtivista. A administração do tempo é uma competência importante, mas ainda é vista como componente desta lógica produtivista pelo enfermeiro, aparecendo apenas como uma forma de racionalizar o trabalho, em busca de melhores desempenhos individuais e $\operatorname{coletivos}^{(7)}$.

A prática profissional do enfermeiro tem evidenciado que sua atuação tende a ser satisfatória na assistência e frágil nas questões de gerenciamento de Enfermagem ou o inverso. Isto mostra que este profissional ainda hoje apresenta dificuldades em articular a gerência e a assistência. $O$ enfermeiro que atua na gestão considera essa dimensão como uma forma de apoiar o cuidado e, ao contrário, aquele que atua diretamente no cuidado inclina-se a menosprezála, rotulando a dimensão gerencial como um serviço burocrático ${ }^{(17)}$.

Vale considerar que o procedimento técnico na assistência, permeado pelo planejamento de ações, pode garantir ao paciente o cuidado de que ele necessita. Quando o enfermeiro planeja, ele determina os objetivos a serem alcançados na promoção do cuidado(17). Mesmo assim, os enfermeiros apresentam grande potencial para articular os serviços de saúde, principalmente os serviços de Enfermagem ${ }^{(20)}$. Este profissional possui competência técnica no exercício profissional, mas se mostra frágil politicamente, isso limita sua autonomia profissional e o subordina a outros profissionais $^{(21)}$. 
Nessa situação é importante que a gerência de Enfermagem da instituição, como líder da equipe de Enfermagem, perceba estes fatores e outros dificultadores, a fim de propiciar formas de amenizar essas e proporcionar melhores condições de trabalho e como consequência melhorar o desempenho dos profissionais, por meio do cuidado eficaz e seguro ao paciente e família ${ }^{(22)}$.

Vale ressaltar que, para que os conhecimentos, habilidades e atitudes para o gerenciamento sejam apreendidos durante a graduação, tem-se evidenciado junto a estudantes de enfermagem que as várias formas dos processos de ensino e aprendizado precisam de reavaliação. Principalmente, no que diz respeito à dissociação da teoria com a prática, mudanças nas instituições formadoras e nas instituições de saúde que constituem os campos das práticas e na relevância em se considerar a percepção dos estudantes, de professores e de profissionais do serviço envolvidos na formação ${ }^{(19)}$.

Como limitação, aponta-se que a pesquisa debruçou-se sobre uma realidade em que os estudantes vivenciaram um contexto de formação específico, o que limita a generalização dos seus resultados. Considerando a dimensão do tema, sugere-se a realização de pesquisas que apresentem inovações na formação para o gerenciamento em enfermagem.

\section{CONSIDERAÇÕES FINAIS}

Os discursos enfatizam o uso de instrumentos gerenciais pelo enfermeiro para o gerenciamento de recursos humanos, materiais, físicos e ambientais, assim como para o gerenciamento do cuidado de Enfermagem, que está localizado em segundo plano.

A pesquisa apontou que o enfermeiro se preocupa em adquirir habilidades gerenciais, visando a cumprir tarefas, ainda sem reflexão e planejamento. Os conhecimentos gerenciais são utilizados em diversas situações, com conhecimentos específicos mobilizados à medida que são requeridos, principalmente no que tange à necessidade da tomada de decisão.

A situação crítica concentra-se no âmbito das atitudes e exige reflexões no sentido de desencadear mudanças prementes, como as relacionadas com a liderança adotada pelos enfermeiros. Espera-se que esta pesquisa possa contribuir para o desenvolvimento da profissão, pois apresenta uma visão geral dos egressos sobre as competências do enfermeiro no mundo do trabalho, principalmente na dimensão gerencial.

As disciplinas presenciadas na graduação sustentam o desenvolvimento de competências gerenciais na prática profissional e impactam na atuação gerencial dos egressos. Mas, outra observação que se faz a respeito dos discursos é relacionada aos conhecimentos, às habilidades e às atitudes para o gerenciamento em Enfermagem. Os dados apontaram que existe maior dificuldade no campo atitudinal das competências gerenciais. Este fato demonstra que a construção das competências gerenciais se inicia na formação, mas o seu desenvolvimento se dá na prática profissional, desmentindo o discurso de que são desenvolvidas na formação.

\section{REFERÊNCIAS}

1. Peres AM, Ciampone MHT. Gerência e competências gerais do enfermeiro. Texto Contexto Enferm. [Internet] 2006;15(3) [acesso 09 ago 2012]. Disponível: http://dx.doi.org/10.1590/S0104-07072006000300015

2. Manenti A, Ciampone MHT, Vera Lucia Mira VL, Minami LF, Soares JMS. The construction process of managerial profile competencies for nurse coordinators in the hospital field. Rev Esc Enferm. USP [Internet]. 2012;46(3)p. 727-33.

3. Zarifian P. Objetivo competência: por uma nova lógica. São Paulo: Atlas; 2001.

4. Ministério da Educação (BR). Conselho Nacional de Educação. Câmara de Educação Superior. Resolução CNE/ CES n. 3, de 7 novembro de 2001. Institui as diretrizes curriculares nacionais do curso de graduação em Enfermagem. Diário Oficial da República Federativa do Brasil. Brasília (DF); 2001. Seção 1, p. 37.

5. Jorge MSB, Freitas CHA, Nóbrega MFB, Queiroz MVO. Gerenciamento em enfermagem: um olhar crítico sobre o conhecimento produzido em periódicos brasileiros (2000-2004). Rev. bras. enferm. Brasília. [Internet] 2007;60(1) [acesso 01 mai 2010]. Disponível: http://www.scielo.br/scielo.php?script=sci_ arttext\&pid=S0034-71672007000100015

6. Tronchin DMR, Gonçalves VLM, Leite MMJ, Melleiro MM. Instrument of student assessment based on nursing managerial competences. Acta paul. enferm. [Internet] 2008; 21(2) [Acesso 02 jun 2010]. Disponível: http://dx.doi.org/10.1590/S0034-71672007000100015 
7. Peres AM, Montezeli JH, Kalinowski CE, Cunha ICKO. Competências gerenciais do enfermeiro. In: Programa de Atualização em Enfermagem (PROENF): Gestão/ Organizadora-geral: Vale EG (org.) Porto Alegre: Artmed/ Panamericana; 2011.

8. Lefevre F, Lefevre AMC. Depoimentos e discursos: uma proposta de análise em pesquisa social. (Série Pesquisa, 12). Brasília: Liber Livro; 2005.

9. Lefevre F, Lefevre AMC. O discurso do sujeito coletivo: um novo enfoque em pesquisa qualitativa. Caxias do Sul: Educs; 2003. (Desdobramentos).

10. Lefevre F, Levefre AMC. O sujeito coletivo que fala. Rev. Interface. [Internet] 2006;10(20) [acesso 03 mar 2010]. Disponível: http://dx.doi.org/10.1590/S141432832006000200017

11. Gaidzinski RR, Leite MMJ, Takahashi RT. O ensino da administração em enfermagem: percepção diante da vivência profissional. Rev Esc Enferm USP. [Internet] 1998;32(1) [acesso 09 abr 2009]. Disponível: http:// dx.doi.org/10.1590/S0080-62341998000100007

12. Luvisotto MM, Vasconcelos AC, Sciarpa LC, Carvalho R. Atividades assistenciais e administrativas do enfermeiro na clínica médico-cirúrgica. Rev. Einstein. [Internet] 2010;8 (2 Pt 1) [acesso 17 ago 2011]. Disponível: http://apps.einstein.br/revista/arquivos/ PDF/1354-Einsteinv8n2_AO1354.pdf

13. Almeida ML, Hexsel SML, Maftum MA, Labronici LM, Peres AM. Instrumentos gerenciais utilizados na tomada de decisão do enfermeiro no contexto hospitalar. Texto contexto - enferm. [Internet]. 2011;20(esp.) [acesso 25 mar 2014]. Disponível: http://dx.doi.org/10.1590/S010407072011000500017

14. Alves VL. Competências essenciais à liderança na Enfermagem quanto ao enfoque de gestão de pessoas. In: Malagutti W, Caetano KC. Gestão do Serviço de Enfermagem no mundo globalizado. Rio de Janeiro: Editora Rubio; 2009.

15. Boccato SBG. Como gerenciar um grupo em conflito. In: Malagutti W, Caetano KC. Gestão do Serviço de Enfermagem no mundo globalizado. Rio de Janeiro: Editora Rubio; 2009.

16. Souza e Souza LP, Cordeiro ALC, Aguiar RN, Santana JMF, Dias OV, et al. A liderança na visão de enfermeiros líderes. Enfermería Global, [Internet] 2013;30 [acesso 09 set 2013]. Disponível: http://revistas.um.es/eglobal/ article/download/eglobal.12.2.154801/147301

17. Hausmann M, Peduzzi M. Articulação entre as dimensões gerencial e assistencial do processo de trabalho do enfermeiro. Texto Contexto Enferm. [Internet] 2009;18(2) [acesso 03 set 2013]. Disponível: http://www.scielo.br/pdf/tce/v18n2/08.pdf
18. Nosow V, Puschel VAA. The teaching of attitudinal content in higher education of nursing. Rev EsC Enferm USP. [Internet] 2009;43(esp.2) [acesso 25 set 2010]. Disponível: http://dx.doi.org/10.1590/S008062342009000600015

19. Llapa-Rodríguez EO, Carvalho TS, Gois CFL, Guimarães AMDN. Vivências dos estudantes de pré-grau com as matérias de administração de enfermagem. Invest Educ Enferm. [Internet] 2012;30(1):87-94. [acesso 07 out 2013]. Disponível: http://aprendeenlinea.udea.edu.co/ revistas/index.php/iee/article/view/9485/10520

20. Berti HW, Braga EM, Godoy I, Spiri WC, Bocchi SCM. . Movement undertaken by newly graduated nurses towards the strengthening of their professional autonomy and towards patient autonomy. Rev. LatinoAm. Enfermagem. [Internet] 2008;16(2) [acesso 4 ago 2009]. Disponível: http://dx.doi.org/10.1590/S010411692008000200003

21. Lopes MMB, Carvalho JN, Backes MTS, Erdmann $\mathrm{AL}$, Meirelles BHS. Políticas e tecnologias de gestão em serviços de saúde e de enfermagem. Acta Paul. Enferm. [Internet] 2009;22(6) [acesso 25 mai 2010]. Disponível: http://dx.doi.org/10.1590/S010321002009000600015

22. Aguiar DF, Conceição-Stipp MA, Leite JL, Mattos VZ, Andrade KBS. Gerenciamento de enfermagem: situações que facilitam ou dificultam o cuidado na unidade coronariana. Aquichán [Internet]. 2010;10(2) [acesso 19 jul 2013]. Disponível: http://www.scielo. org.co/pdf/aqui/v10n2/v10n2a03.pdf 